\title{
AN IMPROVED BOUND FOR THE STAR DISCREPANCY OF SEQUENCES IN THE UNIT INTERVAL
}

\author{
Gerhard Larcher — Florian Puchhammer \\ Dedicated to the memory of Professor Pierre Liardet
}

\begin{abstract}
It is known that there is a constant $c>0$ such that for every sequence $x_{1}, x_{2}, \ldots$ in $[0,1)$ we have for the star discrepancy $D_{N}^{*}$ of the first $N$ elements of the sequence that $N D_{N}^{*} \geq c \cdot \log N$ holds for infinitely many $N$. Let $c^{*}$ be the supremum of all such $c$ with this property. We show $c^{*}>0.065664679 \ldots$, thereby slightly improving the estimates known until now.
\end{abstract}

Communicated by Werner Georg Nowak

\section{Introduction and statement of the result}

Let $x_{1}, x_{2}, \ldots$ be a point sequence in $[0,1)$. By $D_{N}^{*}$ we denote the star discrepancy of the first $N$ elements of the sequence, i.e.,

$$
D_{N}^{*}=\sup _{x \in[0,1]}\left|\frac{\mathcal{A}_{N}(x)}{N}-x\right|,
$$

where $\mathcal{A}_{N}(x):=\#\left\{1 \leq n \leq N \mid x_{n}<x\right\}$. The sequence $x_{1}, x_{2}, \ldots$ is uniformly distributed in $[0,1)$ if and only if $\lim _{N \rightarrow \infty} D_{N}^{*}=0$.

In 1972 W. M. Schmidt [7] showed that there is a positive constant $c$ such that for all sequences $x_{1}, x_{2}, \ldots$ in $[0,1)$ we have

$$
D_{N}^{*}>c \cdot \frac{\log N}{N}
$$

2010 Mathematics Subject Classification: 11K38, 11K06.

Keywords: uniform distribution, discrepancy.

The authors are supported by the Austrian Science Fund (FWF), Project F5507-N26, which is a part of the Special Research Program "Quasi-Monte Carlo Methods: Theory and Applications". 


\section{GERHARD LARCHER — FLORIAN PUCHHAMMER}

for infinitely many $N$. The order $\frac{\log N}{N}$ is best possible. There are many known sequences for which $D_{N}^{*} \leq c^{\prime} \cdot \frac{\log N}{N}$ holds for all $N$ with an absolute constant $c^{\prime}$. For all necessary details on discrepancy and low-discrepancy sequences see the monographs [2] or [5].

So it makes sense to define the one-dimensional star discrepancy constant $c^{*}$ to be the supremum over all $c$ such that (11) holds for all sequences $x_{1}, x_{2}, \ldots$ in $[0,1)$ for infinitely many $N$. Or, in other words,

$$
c^{*}:=\inf _{\omega} \limsup _{N \rightarrow \infty} \frac{N D_{N}^{*}(\omega)}{\log N},
$$

where the infimum is taken over all sequences $\omega=x_{1}, x_{2}, \ldots$ in $[0,1)$, and where $D_{N}^{*}(\omega)$ denotes the star discrepancy of the first $N$ elements of $\omega$.

The currently best known estimates for $c^{*}$ are

$$
0.0646363 \ldots \leq c^{*} \leq 0.222 \ldots
$$

The upper bound was given by Ostromoukhov [6] (thereby slightly improving earlier results of Faure (see, for example, [1])). The lower bound was given by Larcher [3].

It is the aim of this paper to improve the above lower bound for $c^{*}$. That is, what we prove

TheOREM 1. For the one-dimensional star discrepancy constant we have

$$
c^{*} \geq 0.065664679 \ldots
$$

The idea of the proof follows a method introduced by Liardet [4] which was also used by Tijdeman and Wagner in [8] and by Larcher in [3].

\section{Main ideas and proof of Theorem 1}

We will heavily make use of the idea, the notation, and most of the results used and obtained in 3 . In this paper we extend the analysis carried out in the aforementioned paper. In this section we therefore repeat the most important notation and facts from [3] and explain how we extend the method to prove Theorem 1 .

We consider a finite point set $\mathcal{P}=\left\{x_{1}, x_{2}, \ldots, x_{N}\right\}$ in $[0,1)$ with $N=\left[a^{t}\right]$ for some real $a, 3 \leq a \leq 3.7$, and some $t \in \mathbb{N}$. Further, we divide the index-set $A=\{1,2, \ldots, N\}$ into index-subsets $A_{0}, A_{1}, A_{2}$, where $A_{0}=\left\{1,2, \ldots,\left[a^{t-1}\right]\right\}$, $A_{2}=\left\{\left[a^{t}\right]-\left[a^{t-1}\right]+1, \ldots,\left[a^{t}\right]\right\}$, and $A_{1}=A \backslash\left(A_{0} \cup A_{2}\right)$. 


\section{STAR DISCREPANCY OF SEQUENCES}

For simplicity, let us first of all assume that $a^{t}$ and $a^{t-1}$ are integers (of course this can only happen if $a=3$ ). For $x \in[0,1)$ we consider the discrepancy function $D_{n}(x):=\#\left\{i \leq n \mid x_{i}<x\right\}-n x=\mathcal{A}_{n}(x)-n x$ and we define the function $f(x):=\max _{n \in A_{2}} D_{n}(x)-\max _{n \in A_{0}} D_{n}(x)$.

In [3] it was shown that the function $f$ has the following properties:

(i) $f(0)=f(1)=0$.

(ii) $f$ is piecewise linear, piecewise monotonically decreasing, and $|f|$ is bounded by $a^{t}$.

(iii) $f$ is left-continuous and each discontinuity constitutes a positive jump.

(iv) The slope of $f$ is always between $-a^{t}$ and $s_{0}:=-a^{t-1}(a-2)$.

(v) If $f$ is continuous on $[x, y]$ then the slope of $f(x)$ and $f(y)$ can differ at most by $a^{t-1}$.

(vi) $f$ has discontinuities with a jump of height at least 1 in all points $x_{i}$ with $i \in A_{1}$.

Further it was shown in [3, Lemma 2.11] that for given $a$ and $t$ there exists a function $f_{\text {strong }}^{*}:[0,1] \rightarrow \mathbb{R}$ satisfying (i)-(vi) for some $x_{1}, \ldots, x_{N}$ (we say $f_{\text {strong }}^{*}$ is strongly admissible) such that

$$
\int_{0}^{1}\left|f_{\text {strong }}^{*}(x)\right| \mathrm{d} x=\min _{g \text { strongly admissible }} \int_{0}^{1}|g(x)| \mathrm{d} x,
$$

and (in [3, Lemma 2.14]) that for every $\varepsilon>0$ and (now arbitrary) $a \in[3,4]$ and $t$ with $t \geq t(\varepsilon)$

$$
\int_{0}^{1}\left|f_{\text {strong }}^{*}(x)\right| \mathrm{d} x \geq \frac{(a-2)(8 a+3)}{8(1-2 a)^{2}}-\varepsilon .
$$

Finally, we finished the proof of the Theorem in [3] in the following way:

It was shown that (see Section 3 in [3])

$$
\begin{aligned}
\int_{0}^{1}\left(\max _{n \in A} D_{n}(x)-\min _{n \in A} D_{n}(x)\right) \mathrm{d} x & \geq t \int_{0}^{1}\left|f_{\text {strong }}^{*}(x)\right| \mathrm{d} x \\
& \geq t\left(\frac{(a-2)(8 a+3)}{8(1-2 a)^{2}}-\varepsilon\right) \\
& \geq \frac{\log N}{\log a} \cdot\left(\frac{(a-2)(8 a+3)}{8(1-2 a)^{2}}-\varepsilon\right) \\
& \geq 2 \log N \cdot 0.0646363 \ldots
\end{aligned}
$$

if we choose $a=3.71866 \ldots$ and $N$ large enough. Hence there exist $x \in[0,1]$ and $n \leq N$ with

$$
D_{n}(x) \geq 0.0646363 \ldots \cdot \log N
$$

and Theorem 1.1 from [3] follows. 


\section{GERHARD LARCHER — FLORIAN PUCHHAMMER}

To improve the above result from [3] in the present paper we proceed as follows: We show that $f$ has to satisfy an even more restrictive property ( $\left.\mathrm{vi}^{\prime}\right)$ instead of property (vi) and we call a function $g$ satisfying (i)-(v) and (vi') strictly admissible. Moreover, we show that there exists a strictly admissible function $f_{\text {strict }}^{*}:[0,1] \rightarrow \mathbb{R}$ with

and

$$
\int_{0}^{1}\left|f_{\text {strict }}^{*}(x)\right| \mathrm{d} x=\min _{g \text { strictly admissible }} \int_{0}^{1}|g(x)| \mathrm{d} x
$$

$$
\int_{0}^{1}\left|f_{\text {strict }}^{*}(x)\right| \mathrm{d} x \geq \frac{(a-2)\left(12 a+9+(a-2)(4 a-3) \log \left(1+\frac{1}{a-2}\right)\right)}{a\left(a-\frac{1}{2}\right)^{2}\left(3+(a-2) \log \left(1+\frac{1}{a-2}\right)\right)}-\varepsilon
$$

for all $a \in(3,3.7]$ and $t \geq t(\varepsilon)$.

Note that, in the following, we will work with $a^{t}$ and $a^{t-1}$ as if they were integers and we will obtain the above result without " $-\varepsilon$ " and for all $t \geq t_{0}$ in this case. For working with $\left[a^{t-1}\right]$ and $\left[a^{t}\right]$ instead of $a^{t-1}$ and $a^{t}$ we then easily obtain the stated result.

In the very same way as in [3] and as described above we then obtain $D_{n}(x) \geq$ $0.065664679 \ldots \cdot \log N$ for some $x \in[0,1]$ and $n \geq N$ by choosing $a=3.62079 \ldots$ Consequently, Theorem 1 follows.

So it remains to prove the two main auxiliary results, namely, that a stronger property ( $\left.\mathrm{vi}^{\prime}\right)$ for $f$ as well as the lower bound for $\int_{0}^{1}\left|f_{\text {strict }}^{*}(x)\right| \mathrm{d} x$ as stated above hold. This is carried out in the next section. For the proofs of these two results we will have to use some facts already obtained in [3], again.

\section{Proof of the auxiliary results}

Lemma 1. Let $j \in A_{2}$, i.e., $j=a^{t}-a^{t-1}+k$ for some integer $k, 1 \leq k<a^{t-1}$, and assume that $f(x)=\max _{n \in A_{2}} D_{n}(x)-\min _{n \in A_{0}} D_{n}(x)$ has a discontinuity in $x_{j}$. Let further $l_{j}, r_{j} \in A$ such that $\mathcal{P} \cap\left(x_{l_{j}}, x_{r_{j}}\right)=\left\{x_{j}\right\}$. If there exists an $\bar{x} \in\left(x_{j}, x_{r_{j}}\right)$ such that, in $\bar{x} f$ has slope $s(\bar{x})>s_{0}-k$, then $f(\underline{x}) \geq f(\bar{x})-s_{0}(\bar{x}-\underline{x})$ for all $\underline{x} \in\left[x_{l_{j}}, x_{j}\right)$. Here, again, $s_{0}=a^{t-1}(a-2)$ as defined in property (iv) above.

REMARK. The meaning of Lemma 1 is illustrated in Figure 1 . Using the same notation $f(\underline{x})$ lies above the line with slope $s_{0}$ reaching back from the point $(\bar{x}, f(\bar{x}))$ (dashed) in case the slope of $f$ (solid) becomes flatter than $s_{0}-k$. 


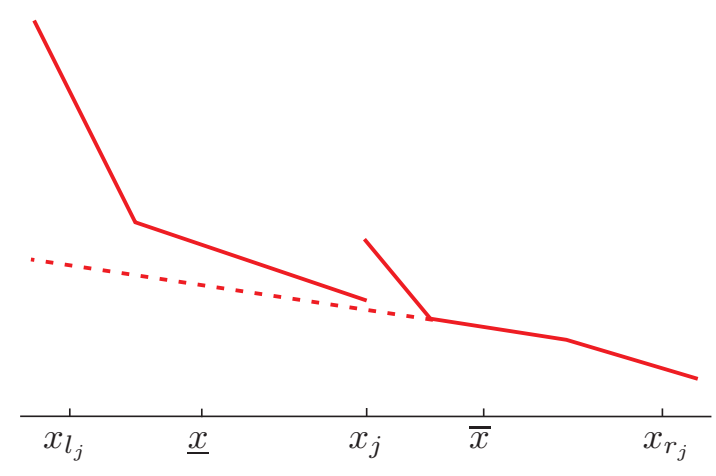

FiguRE 1.

Pro of of Lem ma $\mathbb{1}$. Let $\underline{x}, \bar{x}$ be like above with $s(\bar{x})>s_{0}-k$. We set

$$
\bar{n}_{i}=n_{i}(\bar{x}) \quad \text { and } \quad \underline{n}_{i}=n_{i}(\underline{x})
$$

such that

$$
D_{\bar{n}_{i}}(\bar{x})=\max _{n \in A_{i}} D_{n}(\bar{x}) \quad \text { and } \quad D_{\underline{n}_{i}}(\underline{x})=\max _{n \in A_{i}} D_{n}(\underline{x}) .
$$

So

$$
f(\bar{x})=D_{\bar{n}_{2}}(\bar{x})-D_{\bar{n}_{0}}(\bar{x}) \quad \text { and } \quad f(\underline{x})=D_{\underline{n}_{2}}(\underline{x})-D_{\underline{n}_{0}}(\underline{x}) .
$$

First we show that $\bar{n}_{2}<j$. Indeed, we have

$$
a^{t-1}-\bar{n}_{2} \geq \bar{n}_{0}-\bar{n}_{2}=s(\bar{x})>s_{0}-k=-a^{t-1}(a-2)-k .
$$

Thus, $\bar{n}_{2}<a^{t}-a^{t-1}+k=j$.

Since $\mathcal{A}_{n}$ does not change its value in $x_{j}$ for $n<j, D_{\bar{n}_{2}}$ does not have a jump in $x_{j}$. Consequently, $D_{\bar{n}_{2}}(\bar{x})=D_{\bar{n}_{2}}(\underline{x})-\bar{n}_{2}(\bar{x}-\underline{x})$. This observation yields

$$
D_{\underline{n}_{2}}(\underline{x})-D_{\bar{n}_{2}}(\bar{x}) \geq D_{\bar{n}_{2}}(\underline{x})-D_{\bar{n}_{2}}(\bar{x})=\bar{n}_{2}(\bar{x}-\underline{x}) .
$$

By the same argument we additionally obtain

$$
D_{\underline{n}_{0}}(\underline{x})-D_{\bar{n}_{0}}(\bar{x}) \leq D_{\underline{n}_{0}}(\underline{x})-D_{\underline{n}_{0}}(\bar{x})=\underline{n}_{0}(\bar{x}-\underline{x}) .
$$

Alltogether

$$
\begin{aligned}
f(\underline{x})-f(\bar{x}) & =\left(D_{\underline{n}_{2}}(\underline{x})-D_{\bar{n}_{2}}(\bar{x})\right)-\left(D_{\underline{n}_{0}}(\underline{x})-D_{\bar{n}_{0}}(\bar{x})\right) \\
& \geq\left(\bar{n}_{2}-\underline{n}_{0}\right)(\bar{x}-\underline{x}) \geq-s_{0}(\bar{x}-\underline{x})
\end{aligned}
$$

and the result follows. 


\section{GERHARD LARCHER — FLORIAN PUCHHAMMER}

In addition to the new property of $f$ obtained in Lemma 1 one can easily convince oneself that $f$ is continuous at $x_{1}$. This result is not very efficient yet but nice for calculation purposes. We will use this fact in the following concept of strict admissibility.

Definition 1. A function $g:[0,1] \rightarrow \mathbb{R}$ is called strictly admissible if it satisfies conditions (i) $-(\mathrm{v})$ and the following additional condition $\left(\mathrm{vi}^{\prime}\right)$.

There exists a set $\Gamma=\left\{\xi_{1}, \xi_{2}, \ldots, \xi_{a^{t}-1}\right\} \subset[0,1)$ such that:

a) If $g$ has a jump in $\xi$ then $\xi \in \Gamma$.

b) There exists a set $\Gamma_{1} \subset \Gamma,\left|\Gamma_{1}\right|=a^{t-1}(a-2)$, such that $f$ has a jump of height at least one in each $\xi \in \Gamma_{1}$.

c) There exist $a^{t-1}-1$ further points $\left\{\xi_{k_{1}}, \xi_{k_{2}}, \ldots, \xi_{k_{a^{t-1}-1}}\right\}=: \Gamma_{2}$ with the following property: For each $1 \leq n<a^{t-1}$ let $\xi_{l_{n}}, \xi_{r_{n}} \in \Gamma \cup\{0,1\}$ such that $\Gamma \cap\left(\xi_{l_{n}}, \xi_{r_{n}}\right)=\left\{\xi_{k_{n}}\right\}$. Now, if there is an $\bar{x} \in\left(\xi_{l_{k}}, \xi_{r_{k}}\right)$ with

$$
s(\bar{x})>s_{0}-n
$$

then

$$
g(\underline{x}) \geq g(\bar{x})-s_{0}(\bar{x}-\underline{x})
$$

for all $\underline{x} \in\left[\xi_{l_{n}}, \xi_{k_{n}}\right)$. Here, $s(x)$ denotes the slope of $g$ in $x$.

From the paper [3] and from Lemma 1 it follows that $f$ is strictly admissible. The space of strictly admissible functions, again, is obviously closed with respect to pointwise convergence. Hence, there exists $f_{\text {strict }}^{*}$ strictly admissible with

$$
\int_{0}^{1}|f(x)| \mathrm{d} x \geq \min _{g \text { strictly admissible }} \int_{0}^{1}|g(x)| \mathrm{d} x=\int_{0}^{1}\left|f_{\text {strict }}^{*}(x)\right| \mathrm{d} x .
$$

We intend to estimate $\int_{0}^{1}\left|f_{\text {strict }}^{*}(x)\right| \mathrm{d} x$ from below. To this end we have to derive some properties of $f_{\text {strict }}^{*}$.

Lemma 2. Let $f_{\text {strict }}^{*}$ have a discontinuity in $\gamma$. Then there exist two zeros $\alpha$, $\beta$ of $f_{\text {strict }}^{*}$ with $\alpha<\gamma<\beta$ such that $\gamma$ is the only discontinuity in the interval $(\alpha, \beta)$.

P r o of. First of all, if $\gamma$ is the only point at which $f_{\text {strict }}^{*}$ has a jump, the claim is fulfilled with $\alpha=0$ and $\beta=1$. Hence it suffices to show the following statement: Let $f_{\text {strict }}^{*}$ have two successive discontinuities in, say, $a_{1}$ and $a_{2}, 0<a_{1}<a_{2}<1$. Then $f_{\text {strict }}^{*}$ has a zero in the interval $\left(a_{1}, a_{2}\right)$.

For contradiction we assume $f_{\text {strict }}^{*}>0$ on $\left(a_{1}, a_{2}\right)$ (the case $f_{\text {strict }}^{*}<0$ can be treated quite similarly). In what follows, we will construct a strictly admissible function $\tilde{f}$ such that 


\section{STAR DISCREPANCY OF SEQUENCES}

$$
\int_{0}^{1}|\tilde{f}(x)| \mathrm{d} x<\int_{0}^{1}\left|f_{\text {strict }}^{*}(x)\right| \mathrm{d} x,
$$

which clearly contradicts the definition of $f_{\text {strict }}^{*}$.

Naturally, we need to take special care in constructing $\tilde{f}$ if either $a_{1} \in \Gamma_{2}$ or $a_{2} \in \Gamma_{2}$ which was defined in Definition 1 Moreover, if we manage to preserve the height of any existing jump in any other case then (vi'.b) is automatically fulfilled for $\tilde{f}$.

First of all, we notice that $f_{\text {strict }}^{*}$ cannot have a bend at, say, $y \in\left(a_{1}, a_{2}\right)$ such that the slope before the bend is greater than afterwards. We say $f_{\text {strict }}^{*}$ has a bend in $y$ if $f_{\text {strict }}^{*}$ is continuous in $y$ and if it changes its slope in $y$. Indeed, let $\delta>0$ such that the slope of $f_{\text {strict }}^{*}$ is constant on $[y-\delta, y)$ as well as on $(y, y+\delta]$. Then, as can be seen in Figure 2, we may interchange those pieces such that the resulting function $\tilde{f}$ (solid) remains continuous in $[y-\delta, y+\delta]$. Its absolute integral, however, is smaller than that of $f_{\text {strict }}^{*}$ (dashed). Thus, we need only

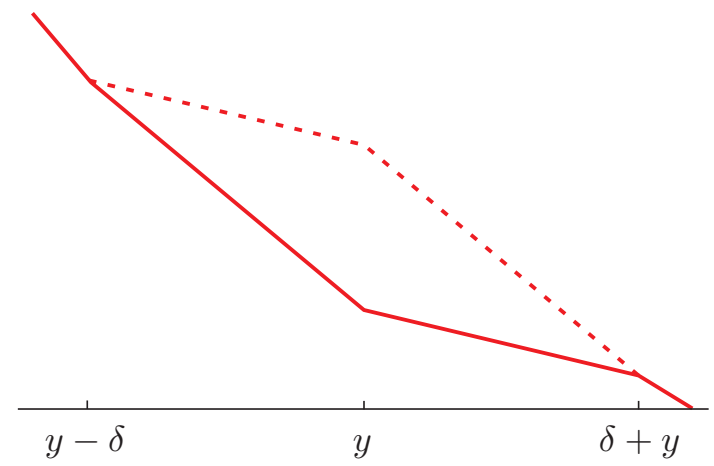

FiguRE 2.

consider bends where $f_{\text {strict }}^{*}$ becomes flatter.

Let now $a_{2} \notin \Gamma_{2}$. We choose $\delta_{1}>0$ such that the slope of $f_{\text {strict }}^{*}$ is a constant $s_{1}$ on $\left(a_{2}, a_{2}+\delta_{1}\right)$. Furthermore, we set

$$
s=\min \left\{s^{*}(x): x \in\left(a_{1}, a_{2}+\delta_{1}\right)\right\},
$$

where $s^{*}$ denotes the slope of $f_{\text {strict }}^{*}$ and where we define $s^{*}\left(a_{2}\right)$ as its left limit. Now, let $0<\delta \leq \min \left\{-2 f_{\text {strict }}^{*}\left(a_{2}\right) /\left(s_{1}+s\right), \delta_{1}\right\}$. With this choice of $\delta$ we have

$$
f_{\text {strict }}^{*}\left(a_{2}\right)+s \delta>-f_{\text {strict }}^{*}\left(a_{2}+\delta\right) \text {. }
$$

In this case we may thus construct $\tilde{f}$ by moving the discontinuity to $\tilde{a}_{2}=a_{2}+\delta$. The missing part of $\tilde{f}$ on the left of $\tilde{a}_{2}$ of length $\delta$ is then chosen such that $\tilde{f}$ 


\section{GERHARD LARCHER — FLORIAN PUCHHAMMER}

is continuous in $a_{2}$ and such that it has constant slope $s$. This construction is visualized in Figure 3 (again $f_{\text {strict }}^{*}$ is represented by the dashed and $\tilde{f}$ by the solid line). This choice for the slope guarantees that the height of the jump is preserved and, additionally, property (vi'.c) from Definition 11 too, cannot be violated by this construction if $a_{1} \in \Gamma_{2}$.

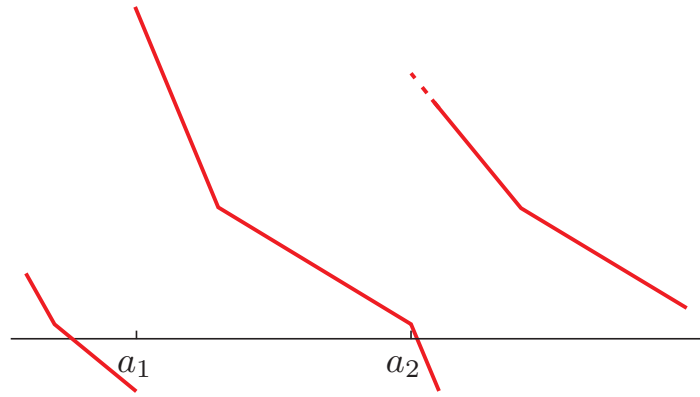

FIGURE 3 .

Certainly, the same construction also works if $a_{2}=\xi_{k_{n}} \in \Gamma_{2}$ for a suitable $k_{n}$ with $s^{*} \leq-a^{t-1}(a-2)-n$ between $a_{2}$ and the next discontinuity of $f_{\text {strict }}^{*}$.

However, if there is some point $x>a_{2}$ before the next jump of $f_{\text {strict }}^{*}$ with $s^{*}(x)>-a^{t-1}(a-2)-n$ we have to proceed differently. In this case, we keep the discontinuity at $a_{2}$ and take the smallest such $x$, call it $\bar{x}$. We define

$$
\tilde{f}(x):=\left\{\begin{aligned}
s_{0}(\bar{x}-x)+f_{\text {strict }}^{*}(\bar{x}) & \text { if } x \in[\bar{x}-\delta, \bar{x}), \\
s^{*}(\bar{x})(\bar{x}-\delta-x)+\tilde{f}(\bar{x}-\delta) & \text { if } x \in\left[a_{2}, \bar{x}-\delta\right), \\
f_{\text {strict }}^{*}(x) & \text { else, }
\end{aligned}\right.
$$

where $\delta>0$ is such that we still have a positive jump in $a_{2}$. Recall that a discontinuity always constitutes a positive jump, hence this is possible. Figure 4 shows $\tilde{f}$ (solid) as well as $f_{\text {strict }}^{*}$ (dashed) in this case. Notice that, again,

$$
\int_{0}^{1}|\tilde{f}(x)| \mathrm{d} x<\int_{0}^{1}\left|f_{\text {strict }}^{*}(x)\right| \mathrm{d} x
$$

and that (vi'.c) from Definition 1 is not violated for $a_{2}$. Additionally, the condition on $\delta$ guarantees that (vi'.c) is not violated for $a_{1}$ if $a_{1} \in \Gamma_{2}$ either. Moreover, we need not take care of the height of the jump in $a_{2}$, since $\Gamma_{1}$ and $\Gamma_{2}$ are disjoint. The dotted line represents the line with slope $s_{0}$ reaching back from $\left\{\bar{x}, f_{\text {strict }}^{*}(\bar{x})\right\}$ which occurs in Definition 1 . 


\section{STAR DISCREPANCY OF SEQUENCES}

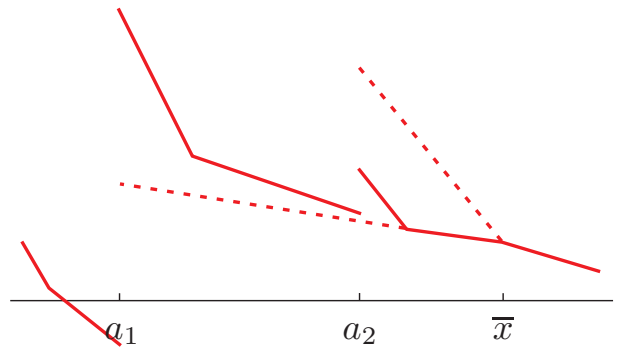

FIGURE 4.

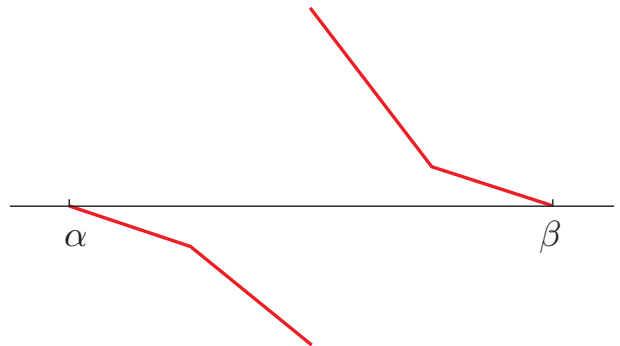

FIGURE 5.

Thus, $f_{\text {strict }}^{*}$ consists of parts $Q$, each of which is defined on an interval $[\alpha, \beta]$ with $f_{\text {strict }}^{*}(\alpha)=f_{\text {strict }}^{*}(\beta)=0$ and such that there is exactly one discontinuity in $(\alpha, \beta)$, see Figure 5 .

In the following we determine the number of such $Q$ 's for $f_{\text {strict }}^{*}$.

LEMma 3. The function $f_{\text {strict }}^{*}$ has exactly $a^{t}-1$ discontinuities.

P r o of. Assume that the total number of discontinuities of $f_{\text {strict }}^{*}$ is less than $a^{t}-1$. Then, in the following, we will define a strictly admissible function $\tilde{f}$ from $f_{\text {strict }}^{*}$ whose absolute integral is smaller than that of $f_{\text {strict }}^{*}$. Let $\Gamma^{*}$ be the set $\Gamma$ from property $\left(\mathrm{vi}^{\prime}\right)$ for the function $f_{\text {strict }}^{*}$.

By assumption there is a $\xi^{*} \in \Gamma^{*}$ such that $f_{\text {strict }}^{*}$ is continuous in $\xi^{*}$. The definition of $\Gamma_{1}^{*}$ (i.e., the set $\Gamma_{1}$ for $f_{\text {strict }}^{*}$ ) guarantees $\xi^{*} \notin \Gamma_{1}^{*}$. Assume that $\xi^{*} \in \Gamma_{2}^{*}$ (the case $\xi^{*} \in \Gamma_{0}^{*}:=\Gamma^{*} \backslash\left(\Gamma_{1}^{*} \cup \Gamma_{2}^{*}\right)$ can be treated quite analogously).

Now choose $\gamma \in \Gamma^{*}$ such that $f_{\text {strict }}^{*}$ has a jump in $\gamma$. We show that $\gamma \in \Gamma_{1}^{*}$ and that $f_{\text {strict }}^{*}$ has a jump of height 1 in $\gamma$ (case $\mathrm{d}$ ) below). Indeed, à priori we are in one of the following four cases:

a) $\gamma \in \Gamma_{0}^{*}$,

b) $\gamma \in \Gamma_{2}^{*}$,

c) $\gamma \in \Gamma_{1}^{*}$ with a jump of height greater than 1 , or

d) $\gamma \in \Gamma_{1}^{*}$ with a jump of height exactly equal to 1 in $\gamma$.

Assume that $\gamma \in \Gamma_{2}^{*}$ (case b). By Lemma $2 \gamma$ is isolated by two successive zeros of $f_{\text {strict }}^{*}$. Hence (3) from property $\left(\mathrm{vi}^{\prime}\right)$ cannot hold, and therefore (2) from the same property does not hold either. Consequently, (see Fig. 6) we can 


\section{GERHARD LARCHER — FLORIAN PUCHHAMMER}

take a point $\tilde{\xi}$ on the left of $\gamma$ and insert a short piece of minimal slope on $[\tilde{\xi}, \gamma)$ without interferring with property (vi'.c). Again, the dashed line represents $f_{\text {strict }}^{*}$ and the solid one the resulting new function $\tilde{f}$. The new set $\tilde{\Gamma}$ is the set $\Gamma^{*}$ with $\xi^{*}$ replaced by $\tilde{\xi}$.

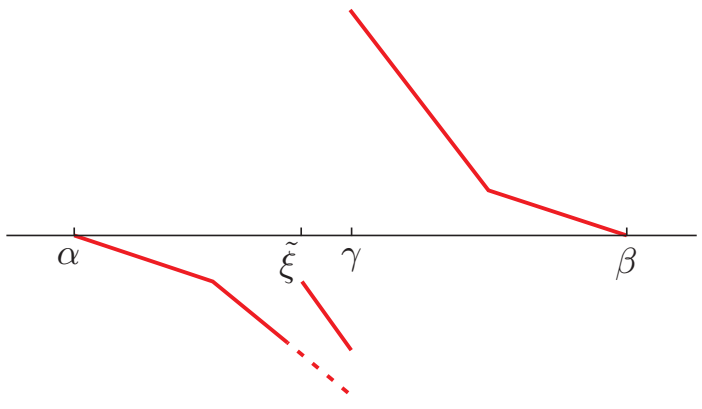

FiguRE 6.

This construction also works for case a) in the same way, and, with some special care, i.e., the jump of $\tilde{f}$ in $\gamma$ maintains a height of at least one, for the case c) too.

Consequently, $f_{\text {strict }}^{*}$ can only have the $a^{t-1}(a-2)$ jumps at the positions given by $\Gamma_{1}^{*}$. All these jumps have height exactly equal to one and there are absolutely no further jumps. Obviously, $f_{\text {strict }}^{*}$ cannot have slope $-a^{t}$ everywhere, since then

$$
0>a^{t-1}(a-2)-a^{t}=f_{\text {strict }}^{*}(1),
$$

a contradiction to property (i). Thus, there exists an interval $\left[\delta_{1}, \delta_{2}\right]$ such that $f_{\text {strict }}^{*}>0\left(\right.$ or $\left.f_{\text {strict }}^{*}<0\right)$ on $\left[\delta_{1}, \delta_{2}\right]$ and its slope is greater than $-a^{t}$. We choose $\delta^{\prime} \in\left(\delta_{1}, \delta_{2}\right)$ sufficiently close to $\delta_{1}$ (or to $\left.\delta_{2}\right)$ and define

or

$$
\tilde{f}(x)=\left\{\begin{aligned}
f_{\text {strict }}^{*}\left(\delta_{1}\right)-a^{t}\left(x-\delta_{1}\right) & \text { if } x \in\left(\delta_{1}, \delta^{\prime}\right], \\
f_{\text {strict }}^{*}(x) & \text { else, }
\end{aligned}\right.
$$

$$
\tilde{f}(x)=\left\{\begin{aligned}
f_{\text {strict }}^{*}\left(\delta_{2}\right)-a^{t}\left(x-\delta_{2}\right) & \text { if } x \in\left(\delta^{\prime}, \delta_{2}\right], \\
f_{\text {strict }}^{*}(x) & \text { else, }
\end{aligned}\right.
$$

respectively. See Figures 7 and 8 .

From the above results we obtain that $f_{\text {strict }}^{*}$ has to be of the following form: It divides $[0,1)$ into $a^{t}-1$ parts $[\alpha, \beta)$ with $f_{\text {strict }}^{*}(\alpha)=f_{\text {strict }}^{*}(\beta)=0$, and $f_{\text {strict }}^{*}$ has exactly one discontinuity $\gamma \in(\alpha, \beta)$. We say that $[\alpha, \beta)$ is of type $Q_{i}$ if $\gamma \in \Gamma_{i}^{*}$ for $i=0,1,2$. 


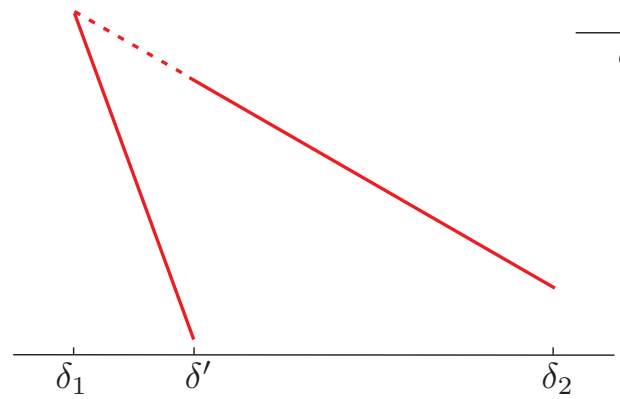

Figure 7 . Case $f_{\text {strict }}^{*}>0$ on $\left[\delta_{1}, \delta_{2}\right] \quad$ Figure 8 . Case $f_{\text {strict }}^{*}<0$ on $\left[\delta_{1}, \delta_{2}\right]$

From [3, the equation (2), we know that, for an interval of type the $Q_{0}$ (this corresponds to the type $Q^{\prime \prime}$ in the abovementioned paper), we have

$$
\int_{\alpha}^{\beta}\left|f_{\text {strict }}^{*}(x)\right| \mathrm{d} x \geq \chi^{2} \frac{a^{t-1}(a-2)}{4}, \quad \chi=\beta-\alpha,
$$

and from [3, Lemma 2.12] and the considerations following the proof of this lemma we know that for an interval of type $Q_{1}$ (this corresponds to the type $Q^{\prime}$ in the abovementioned paper) we have

$$
\int_{\alpha}^{\beta}\left|f_{\text {strict }}^{*}(x)\right| \mathrm{d} x \geq \frac{\chi\left(4-a^{t-1} \chi\right)}{16}, \quad \chi=\beta-\alpha .
$$

Moreover, we know from [3, Lemma 2.10] that for $f_{\text {strict }}^{*}$ all $a^{t-1}$ intervals $Q_{0}$ have the same length and all $a^{t}-2 a^{t-1}$ intervals $Q_{1}$ have the same length.

Lemma 4. For $1 \leq n \leq a^{t-1}-1$ let $Q_{2}^{(n)}$ be given by the interval $[\alpha, \beta)$. Then we have

$$
\int_{Q_{2}^{(n)}}\left|f_{\text {strict }}^{*}(x)\right| \mathrm{d} x \geq(\beta-\alpha)^{2} \frac{\left|s_{0}\right|\left(n+\left|s_{0}\right|\right)}{2\left(n+2\left|s_{0}\right|\right)} .
$$

Pr o of. This follows from the remark preceeding Lemma 4 and simple calculations.

To finish the proof of our theorem we finally show:

Lemma 5. For all $3 \leq a \leq 3.7$ we have

$$
\int_{0}^{1}\left|f_{\text {strict }}^{*}(x)\right| \mathrm{d} x \geq \frac{(a-2)\left(12 a+9+(a-2)(4 a-3) \log \left(1+\frac{1}{a-2}\right)\right)}{16\left(a-\frac{1}{2}\right)^{2}\left(3+(a-2) \log \left(1+\frac{1}{a-2}\right)\right)} .
$$




\section{GERHARD LARCHER — FLORIAN PUCHHAMMER}

P r o of. Due to Lemma 4 and the remarks preceeding it we have to minimize the right hand-side of

$$
\begin{aligned}
\int_{0}^{1}\left|f_{\text {strict }}^{*}(x)\right| \mathrm{d} x \geq & a^{t-1} \cdot \chi_{0}^{2} \frac{a^{t-1}(a-2)}{4}+a^{t-1}(a-2) \cdot \frac{\chi_{1}\left(4-a^{t-1} \chi_{1}\right)}{16} \\
& +\sum_{n=1}^{a^{t-1}-1}\left(\chi_{2}^{(n)}\right)^{2} \frac{\left|s_{0}\right|\left(n+\left|s_{0}\right|\right)}{2\left(n+2\left|s_{0}\right|\right)} \\
= & : a^{t-1} \cdot \chi_{0}^{2} \tilde{A}_{0}+a^{t-1}(a-2) \cdot \frac{\chi_{1}\left(4-a^{t-1} \chi_{1}\right)}{16} \\
& +\sum_{n=1}^{a^{t-1}-1}\left(\chi_{2}^{(n)}\right)^{2} \tilde{A}_{n}
\end{aligned}
$$

with respect to $\chi_{0}, \chi_{1}, \chi_{2}^{(n)} \geq 0$ (these quantities denote the lengths of the intervals $\left.Q_{0}, Q_{1}, Q_{2}^{(n)}\right)$ under the constraint

$$
a^{t-1} \chi_{0}+a^{t-1}(a-2) \chi_{1}+\sum_{n=1}^{a^{t-1}-1} \chi_{2}^{(n)}=1 .
$$

The Lagrangian approach immediately implies

$$
\tilde{A}_{0} \chi_{0}=\tilde{A}_{n} \chi_{2}^{(n)} \quad \text { for all } 1 \leq n<a^{t-1} .
$$

The constraint therefore yields

$$
\chi_{0}=\frac{1-a^{t-1}(a-2) \chi_{1}}{a^{t-1}+\sum_{n=1}^{a^{t-1}-1} \frac{\tilde{A}_{0}}{\tilde{A}_{n}}} .
$$

Moreover, the denominator in the above equation simplifies to

$$
\begin{aligned}
a^{t-1}+\sum_{n=1}^{a^{t-1}-1} \frac{\tilde{A}_{0}}{\tilde{A}_{n}} & =a^{t-1}+\sum_{n=1}^{a^{t-1}-1}\left(1-\frac{n}{2\left(\left|s_{0}\right|+n\right)}\right) \\
& =2 a^{t-1}-1-\frac{1}{2} \sum_{n=\left|s_{0}\right|+1}^{a^{t-1}-1+\left|s_{0}\right|}\left(1-\frac{\left|s_{0}\right|}{n}\right) \\
& =\frac{1}{2}\left(3 a^{t-1}-1+\left|s_{0}\right| \sum_{n=\left|s_{0}\right|+1}^{t-1} \frac{1}{n}\right) .
\end{aligned}
$$




\section{STAR DISCREPANCY OF SEQUENCES}

The latter sum can be bounded by $\log (1+1 /(a-2))$ from above. We summarize our intermediate findings and obtain

$$
\begin{aligned}
\int_{0}^{1}\left|f_{\text {strict }}^{*}(x)\right| \mathrm{d} x \geq & \frac{(a-2)\left(1-a^{t-1}(a-2) \chi_{1}\right)^{2}}{2\left(3+(a-2) \log \left(1+\frac{1}{a-2}\right)\right)} \\
& +a^{t-1}(a-2) \frac{\chi_{1}\left(4-a^{t-1} \chi_{1}\right)}{16}=: p\left(\chi_{1}\right) .
\end{aligned}
$$

Now, our goal is to minimize the function $p$. We immediately see that $p$ is a polynomial of degree two and its leading coefficient is positive for all $3<a \leq 3.7$.

Thus, it attains its minimum at its only critical point

$$
\chi_{\mathrm{crit}}=a^{1-t} \frac{2\left(4 a-11-(a-2) \log \left(1+\frac{1}{a-2}\right)\right)}{29+8 a(a-4)-(a-2) \log \left(1+\frac{1}{a-2}\right)} .
$$

On the other hand, from the proof of Lemma 2.13 in [3] we know that we have the following bounds for $\chi_{1}$

$$
\chi_{\min }:=\frac{a^{1-t}}{a-\frac{1}{2}} \leq \chi_{1} \leq \frac{a^{1-t}}{a-\frac{3}{2}} .
$$

We will show that $\chi_{\text {crit }} \leq \chi_{\text {min }}$. Indeed, it can easily be verified that the denominator of $\chi_{\text {crit }}$ is positive. Thus, $\chi_{\text {crit }}>\chi_{\min }$ if and only if

$$
0>3 a-9-(a-1)(a-2) \log \left(1+\frac{1}{a-2}\right)=: q(a) .
$$

We observe that $q(3.7)<0$ and, additionally, that $q^{\prime}(a)>0$ for all $a \in(3,3.7]$. Hence

$$
\chi_{1}=\frac{a^{1-t}}{a-\frac{1}{2}}
$$

and by inserting this value into the function $p$ the result follows.

\section{REFERENCES}

[1] FAURE, H.: Good permutations for extreme discrepancy, J. Number Theory 42 (1992), no. $1,47-56$.

[2] KUIPERS, L.-NIEDERREITER, H.: Uniform Distribution of Sequences, John Wiley, New York, 1974.

[3] LARCHER, G.: On the star discrepancy of sequences in the unit interval, J. Complexity 31 (2015), no. 3, 474-485.

[4] LIARDET, P.: Discrepance sur le cercle, Primaths I, Univ. Marseille, 1979, 7-1. 


\section{GERHARD LARCHER — FLORIAN PUCHHAMMER}

[5] NIEDERREITER, H.: Random Number Generation and Quasi-Monte Carlo Methods. In: CBMS-NSF Regional Conference Series in Applied Mathematics Vol. 63, SIAM, Philadelphia, 1992.

[6] OSTROMOUKHOV, V.: Recent progress in improvement of extreme discrepancy and star discrepancy of one-dimensional sequences. In: Monte Carlo and quasi-Monte Carlo methods 2008, Springer, Berlin, 2009, pp. 561-572.

[7] SCHMIDT, W. M.: Irregularities of distribution, VII., Acta Arith. 21 (1972), 45-50.

[8] TIJDEMAN, R.-WAGNER, G.: A sequence has almost nowhere small discrepancy, Monatsh. Math. 90 (1980), no. 4, 315-329.

Received December 1, 2014

Accepted October 2, 2015

\section{Gerhard Larcher}

Institute of Financial Mathematics and Applied Number Theory

University Linz

Altenbergerstraße 69

4040 Linz

AUSTRIA

E-mail: gerhard.larcher@jku.at

\section{Florian Puchhammer}

Institute of Financial Mathematics and Applied Number Theory

University Linz

Altenbergerstraße 69

4040 Linz

AUSTRIA

E-mail: florian.puchhammer@jku.at 